\title{
Diagnostik implantatassoziierter Infektionen und Stellenwert der Sonikation
}

\author{
Susanne Feihl, Andrej Trampuz, Nora Renz
}

\section{Einleitung}

Der Einsatz von Fremdmaterial ist in vielen Bereichen der modernen Medizin etabliert. Die Problematik biofilmassoziierter Infektionen stellt mit zunehmendem Einsatz von Implantaten eine relevante und herausfordernde Komplikation dar. Die Infektionsrate liegt nach primärer prothetischer Gelenkversorgung bei 1-3\% und nach osteosynthetischer Frakturversorgung bei $1-5 \%[6,10]$. Nach offenen Frakturen liegt die Infektionsrate mit bis zu 30\% noch deutlich höher [13]. Die frühe Diagnose einer Infektion ist für die weiteren Therapieschritte und den Behandlungserfolg entscheidend. In der Orthopädie und Unfallchirurgie wurde durch die Etablierung diagnostischer Kriterien ( $\bullet$ Tab.1) und Entwicklung diagnostischer Algorithmen die Sicherung der Infektionsdiagnose erleichtert. Im Folgenden wird das Vorgehen bei Verdacht auf eine fremdmaterialassoziierte Infektion erläutert.

\section{Anamnese und Klinik}

Persistierende Schmerzen, vor allem in Ruhe oder nachts, deuten auf eine Infektion hin und müssen durch weitere diagnostische Tests überprüft werden. Die Verabreichung von Antibiotika sowie eine prolongierte Sekretion nach der Indexoperation oder Revisionseingriffe sind weitere Hinweise für eine implantatassoziierte Infektion.

Die Ausprägung des klinischen Befunds wird durch mehrere Faktoren, wie chirurgische Versorgung, Lokalisation der Infektion, Infektionspathogenese, Pathogenität des verursachenden Erregers und Zeitpunkt des Auftretens nach der Indexoperation, beeinflusst. Lokale klinische Symptome, wie Rötung, Schwellung und Überwärmung, zeigen sich, abhängig von den umgebenden Weichteilen, in unterschiedlich starker Intensität. Chronische („lowgrade“) Infektionen werden meist durch niedrig virulente Erreger, wie koagulasenegative Staphylokokken oder Propionibakterien, hervorgerufen und äußern sich oft mit einem dezenten klinischen Untersuchungsbefund. Eindeutige Zeichen einer Infektion sind ein sichtbares Implantat, eine perkutane Fistel oder Eiter um das Implantat.
Akute Infektionen werden i.d.R. durch Erreger wie Staphylococcus aureus oder gramnegative Bakterien verursacht und können früh postoperativ oder durch hämatogene Streuung an den Infektionsort gelangen. Eine postoperativ persistierende Wundsekretion ist Hinweis für eine akute Infektion. Systemische Infektionszeichen, wie Fieber oder Schüttelfrost, sind vorwiegend bei akuter hämatogener Streuung mit primärem Infektionsfokus in einem anderen Organsystem zu finden $[3,10]$.

\section{Laborparameter}

Das C-reaktive Protein (CRP) und die Leukozytenzahl im Blut werden häufig routinemäßig bestimmt und sind bei akuten Infektionen i.d. R. erhöht. Bei „low-grade“-Infektionen - insbesondere bei vorliegender perkutaner Fistel - ist der CRP-Wert oft im Normbereich und somit die Sensitivität nicht ausreichend. Nach einer Operation steigt der wenig spezifische CRP-Wert im Serum an, der postoperative Verlauf kann bei persistierend hohem Wert oder erneutem Anstieg hinweisend für eine frühe postoperative implantatassoziierte Infektion sein. Die Blutsenkungsgeschwindigkeit (BSG) wird aufgrund der schlechteren Sensitivität nicht mehr eingesetzt.

\section{Gelenkpunktion}

\begin{abstract}
Merke
Die Aspiration von Synovialflüssigkeit mit der Zellzahlanalyse ist die aussagekräftigste präoperative Untersuchung zum Nachweis einer Gelenkprotheseninfektion.
\end{abstract}

Eine Leukozytenzahl > 2000/ $\mu$ l oder ein prozentualer Anteil der Granulozyten $>70 \%$ weist mit einer Sensitivität von ca. 90\% und einer Spezifität von ca. 95\% auf eine Infektion hin [11]. Vorherige Antibiotikatherapien beeinflussen die Leukozyten- und Granulozytenzahl nicht. Die Aussagekraft der mikrobiologischen Untersuchung wird hingegen von mehreren Faktoren beeinflusst. So kann nach vorheriger Antibiotikatherapie, aber auch bei „lowgrade“-Infektionen das mikrobiologische Kulturergebnis trotz Infektion negativ ausfallen. Durch die Punktion können nur freie (planktonische) Bakterien in der Gelenkflüssigkeit nachgewiesen werden. Vor allem bei chronischen 
- Tab. 1 Diagnostische Kriterien für die periimplantäre Infektion. Vorliegen einer periprothetischen Infektion, wenn $\geq 1$ der mit * gekennzeichneten Kriterien erfüllt ist. Bei mit ** gekennzeichneten Kriterien handelt es sich um für eine Infektion suggestive Hinweise.

\begin{tabular}{|c|c|c|}
\hline \multirow[t]{2}{*}{ Untersuchung } & Kriterium & \multirow[b]{2}{*}{ Prothese } \\
\hline & Osteosynthese & \\
\hline Anamnese** & \multicolumn{2}{|c|}{$\begin{array}{l}\text { - Ruhe- und Nachtschmerz } \\
\text { " prolongierte Wundsekretion } \\
\text { " vorangehende Revisionen und Antibiotikagaben }\end{array}$} \\
\hline Radiologie** & $\begin{array}{l}\text { - Implantatlockerung } \\
\text { - Infektionskallus } \\
\text { - Sequester } \\
\text { - Osteolysen } \\
\text { - Sklerosierung der Kortikalis } \\
\text { - Pseudarthrose }\end{array}$ & $\begin{array}{l}\text { - Prothesenlockerung } \\
\text { - heterotope Ossifikationen } \\
\text { - Fistelgang* }\end{array}$ \\
\hline Klinik* & \multicolumn{2}{|l|}{$\begin{array}{l}\text { - Fistel } \\
\text { - eitrige Wundsekretion } \\
\text { - sichtbares Implantat } \\
\text { " „positive probe-to-implant“ }\end{array}$} \\
\hline Histologie* & $\begin{array}{l}\text { Infektnachweis im periimplantären Gewe- } \\
\text { be, falls }>5 \text { Granulozyten pro Gesichtsfeld } \\
\text { bei } 400 \text {-facher Vergrößerung }\end{array}$ & $\begin{array}{l}\text { Infektnachweis, falls } \geq 23 \text { Granulozyten in } \\
10 \text { Gesichtsfeldern in } 400 \text {-facher Vergrößerung } \\
\text { (Typ II oder III nach Krenn und Morawietz) }\end{array}$ \\
\hline Zellzahl im Gelenkpunktat* & \multicolumn{2}{|l|}{ 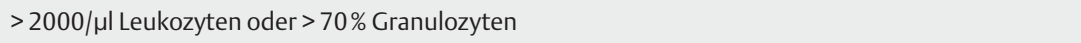 } \\
\hline Mikrobiologie* & \multicolumn{2}{|c|}{$\begin{array}{l}\text { Erregernachweis in: } \\
\text { " } \geq 2 \text { Gewebeproben }{ }^{1} \\
\text { " Sonikationsflüssigkeit ( } \geq 50 \text { Kolonien/ml) }{ }^{2} \\
\text { - Synovialflüssigkeit (bei Gelenkbeteiligung bzw. Prothesen) }\end{array}$} \\
\hline
\end{tabular}

Infektionen adhärieren die Bakterien vorwiegend als Biofilm auf Implantatoberflächen und können in der Gelenkflüssigkeitskultur nur schwer nachgewiesen werden. Eine Verbesserung der Sensitivität der mikrobiologischen Kultur kann durch verlängerte Bebrütung (14 Tage) erreicht werden. Der Nachweis von Bakterien der Hautflora (wie z. B. koagulasenegative Staphylokokken) in der Langzeitbebrütung muss in Zusammenschau mit anderen Diagnostikbausteinen kritisch bewertet und eine Kontamination differenzialdiagnostisch mit in die Überlegung einbezogen werden.

\section{Bildgebung}

Als initiale radiologische Diagnostik wird i.d.R. ein konventionelles Röntgenbild in 2 Ebenen durchgeführt. Bei chronischen Infektionen ist diese einfache bildgebende Methode häufig wegweisend. Zeichen einer Infektion stellen sich z. B. als Lockerung des Implantats, Verbreiterung des Frakturspalts, fehlende Knochenkonsolidierung (Pseudarthrose) oder periimplantäre Osteolysen/Osteophyten dar [3].

In der Computertomografie (CT) mit Kontrastmittel können begleitende Weichteilabszesse, Knochensequester, Pseudarthrosen und eine Lockerung des Implantats dar- gestellt werden. Die Magnetresonanztomografie (MRT) ermöglicht eine Beurteilung der Weichteile oder einer intramedullären Ausbreitung der Infektion (Markraumphlegmone). Artefakte durch Fremdmaterial können sowohl die Beurteilbarkeit im CT als auch im MRT einschränken. Weitere nuklearmedizinische Untersuchungsverfahren, wie z.B. die Positronenemissionstomografie (PET-CT) oder Szintigrafie, können zusätzlich Hinweise über ein infektiöses Geschehen geben, haben aber keinen Stellenwert in der Routinediagnostik der periimplantären Infektion. Die Aussagekraft ist bis zu 24 Monate postoperativ aufgrund lokaler Begleitreaktionen eingeschränkt [3].

\section{Histologische Untersuchung}

Ein sicheres Kriterium für eine Infektion ist eine positive Histologie. Eine Infektion nach ostheosyntetischer Versorgung ist histologisch belegt bei Nachweis von > 5 Granulozyten pro Gesichtsfeld in 400-facher Vergrößerung [7]. Periprothetische Gewebeproben werden histologisch nach Krenn und Morawietz in 4 Typen klassifiziert. Der Nachweis des infektiösen Typs (Typ II) oder des gemischten Typs Abrieb/Infektion (Typ III) spricht für eine periprothetische Infektion [4]. 


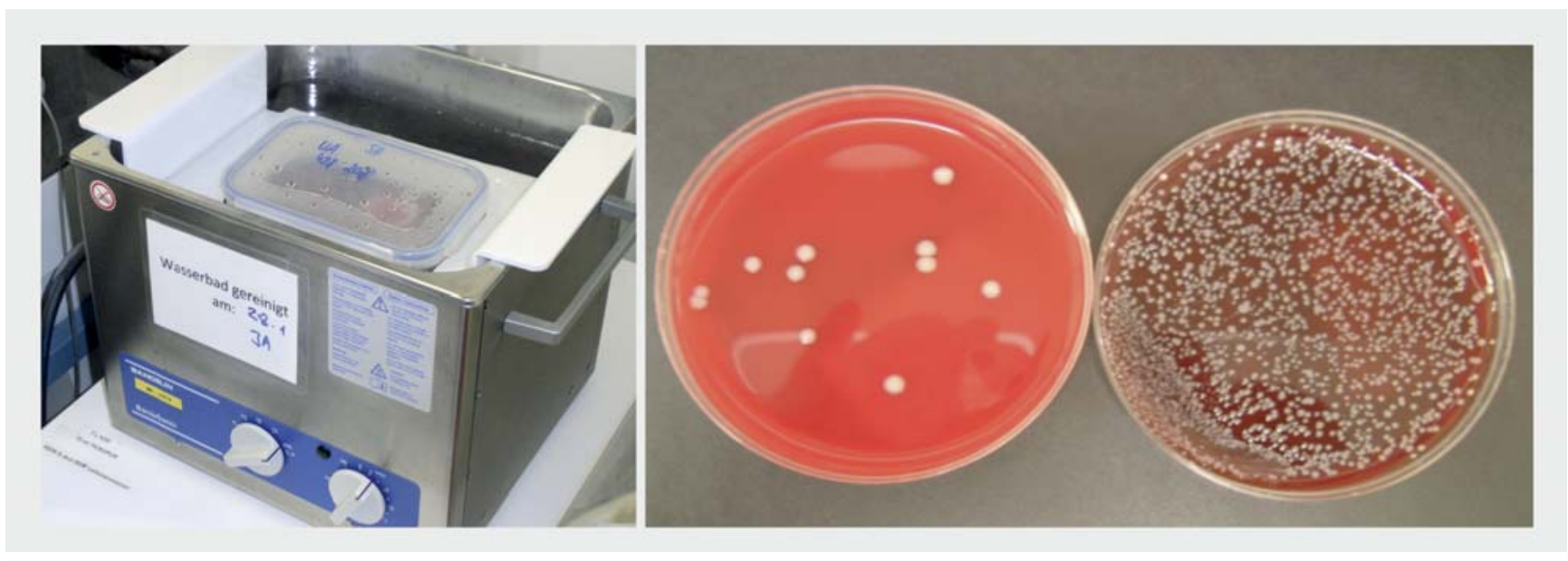

- Abb. 1 Ultraschallbad für die Sonikation; Vergleich Kultur Gewebebiopsie und Sonikationsflüssigkeit.

\section{Mikrobiologische Nachweismethoden}

\section{Merke}

Der kulturelle Nachweis der verursachenden Mikroorganismen und die Bestimmung der Erregerresistenz tragen wesentlich zu einer zielgerichteten und erfolgreichen antibiotischen Therapie bei.

Neben der mikrobiologischen Untersuchung der Synovialflüssigkeit wird die Entnahme von 3 bis 5 periimplantären Gewebebiopsien für die mikrobiologische Kultur und die histologische Analyse empfohlen. Die Probenentnahme in direkter Nähe des Implantats (sog. „Interface“) erhöht die Sensitivität der mikrobiologischen Untersuchung. Die Materialgewinnung in Form von Abstrichen wird aufgrund der niedrigeren Nachweiswahrscheinlichkeit nicht empfohlen [2]. Eine bereits begonnene antibiotische Therapie kann ebenfalls die kulturelle Anzucht der Erreger erschweren und sollte bei klinisch stabilen Patienten 2 Wochen vor Probenahme abgesetzt werden.

Der Nachweis hochvirulenter Erreger, wie Staphylococcus aureus oder Escherichia coli, wird bereits bei kulturellem Wachstum in 1 Probe als relevant gewertet. Demgegenüber sollten niedrigvirulente Erreger, wie Staphylococcus epidermidis oder Propionibacterium acnes, in mindestens 2 Proben nachgewiesen werden, um eine Kontamination auszuschließen.

\section{Stellenwert der Sonikation}

Biofilme entstehen durch die Adhäsion planktonischer Bakterien auf Oberflächen und die Produktion einer Matrix aus polymerisierten extrazellulären Substanzen. In dieser amorphen Matrix eingebettet, befinden sich die Bakterien in einem verminderten metabolischen Zustand und teilen sich nur sehr langsam. Mit den beschriebenen konventionellen mikrobiologischen Verfahren können nur planktonische Bakterien detektiert werden. Insbesondere bei Patienten mit chronischen („low-grade“) Infektionen befinden sich die Erreger in einem „reifen“ Biofilm auf der Implantatoberfläche und die mikrobiologischen Kulturen sind häufig falsch negativ.

Eine Methode, um Biofilme von Oberflächen abzulösen, ist die Sonikation. Implantate werden in einem sterilen Behälter mit steriler Flüssigkeit überschichtet und mit UItraschallwellen behandelt, die sich in der Flüssigkeit ausbreiten und an der Implantatoberfläche zu Mikroströmungen, Scherkräften und oszillierenden Kavitationsblasen führen. Durch die Verwendung eines niedrigfrequenten $(40 \mathrm{kHz})$ und energiearmen $\left(0,2-1 \mathrm{~W} / \mathrm{cm}^{2}\right)$ Ultraschalls werden die Bakterien schonend ohne signifikante Zerstörung von Zellstrukturen vom Fremdmaterial abgelöst [9]. Anschließend wird die resultierende Sonikationsflüssigkeit in der Mikrobiologie weiterverarbeitet und untersucht. Mit diesem Verfahren können bis zu 10000-mal mehr Bakterien als mit den Standardmethoden (Gewebeproben) detektiert werden (Sensitivität 79 vs. 61\%, $p<0,001$, Spezifität 99\%) [12]. Da Bakterien im Biofilm trotz Antibiotikatherapie überleben können, zeigt die Sonikation, insbesondere nach erfolgter antibiotischer Therapie, eine bessere Sensitivität als die Kultur von Gewebebiopsien. Darüber hinaus werden in der Sonikationsflüssigkeit häufiger Mischinfektionen nachgewiesen, die in der konventionellen Gewebeprobe nicht detektiert werden.

Molekularbiologische Methoden, wie die Polymerasekettenreaktion (PCR), lassen eine zusätzliche Verbesserung der Sensitivität erwarten. In klinischen Studien konnte bereits gezeigt werden, dass vor allem bei antibiotisch vorbehandelten Patienten eine bessere Sensitivität erreicht werden kann [1]. Aktuell ist jedoch die PCR noch 
nicht Bestandteil der mikrobiologischen Routinediagnostik.

\section{Durchführung der Sonikation}

Für die Behandlung im Ultraschallbad wird das entfernte Implantat in einem sterilen und wasserdichten Gefäß mit steriler Flüssigkeit (z. B. Ringer-Lösung) zu ca. $90 \%$ bedeckt. Es sollten Plastikbehälter mit verschiedenen Volumina zur Verfügung stehen, und das kleinste Behältnis, welches das Implantat aufnimmt, sollte verwendet werden. Die Transportgefäße werden bei max. $121^{\circ} \mathrm{C}$ für 15 min autoklaviert oder mit Plasmasterilisation sterilisiert. Um den Biofilm vom Implantat zu lösen, wird in einem 1. Schritt der verschlossene Behälter $30 \mathrm{~s}$ von Hand geschüttelt. Anschließend erfolgt eine 1-minütige Beschallung im Ultraschallbad (BactoSonic, Bandelin) mit $40 \mathrm{kHz}$ und $0,2-1 \mathrm{~W} \mathrm{~cm}{ }^{2}$. Durch erneutes Schütteln für $30 \mathrm{~s}$ löst sich der Biofilm und befindet sich in der das Implantat umgebenden Flüssigkeit (Sonikationsflüssigkeit/Sonikat) ( $\bullet$ Abb. 1). Eine festgelegte Menge der Sonikationsflüssigkeit wird auf aerobe und anerobe Agarplatten aufgebracht und in Thioglykolat-Flüssigmedium inokuliert und bebrütet. Die Angabe der Bakterienmenge erfolgt anschließend quantitativ als koloniebildende Einheiten (KBE) pro Milliliter Sonikationsflüssigkeit und ermöglicht eine Interpretation des Ergebnisses hinsichtlich der Relevanz des nachgewiesenen Erregers. Der Nachweis von $\geq 50 \mathrm{KBE} / \mathrm{ml}$ wird als relevant und als Kriterium für eine implantatassoziierte Infektion gewertet. Geringere Keimmengen müssen in Zusammenschau mit den Kulturergebnissen der Gewebebiopsien sowie weiteren Diagnostikbausteinen bewertet werden. In die Bewertung sollten auch immer vorangegangene Antibiotikatherapien mit einbezogen werden. Um langsam wachsende Erreger zu detektieren, ist eine verlängerte Bebrütung der Flüssigmedien für 14Tage empfohlen. Die Beimpfung von Blutkulturflaschen mit Sonikationsflüssigkeit erhöht die Nachweiswahrscheinlichkeit und führt zu einer schnelleren Detektion der Keime [8]. Das Implantat ist Patienteneigentum und muss nach der Sonikation dem Patienten zurückgegeben oder aufbewahrt werden.

\section{Schlussfolgerung}

\footnotetext{
Merke

Für die Diagnose der implantatassoziierten Infektion werden die diagnostischen Kriterien Anamnese, klinisches Erscheinungsbild, Bildgebung, laborchemische Entzündungsparameter, Mikrobiologie und Histologie gemeinsam bewertet.
}

Für die zielgerichtete antibiotische Therapie ist der Erregernachweis Grundvoraussetzung. Dieser ist jedoch bei biofilmassoziierten Infektion häufig erschwert. Eine Ver- besserung der Sensitivität der mikrobiologischen Diagnostik ist durch Anwendung der Sonikation möglich, mit der Biofilme von Implantatoberflächen abgelöst werden können.

\section{Interessenkonflikt}

Kein Interessenskonflikt.

\section{Über die Autoren}

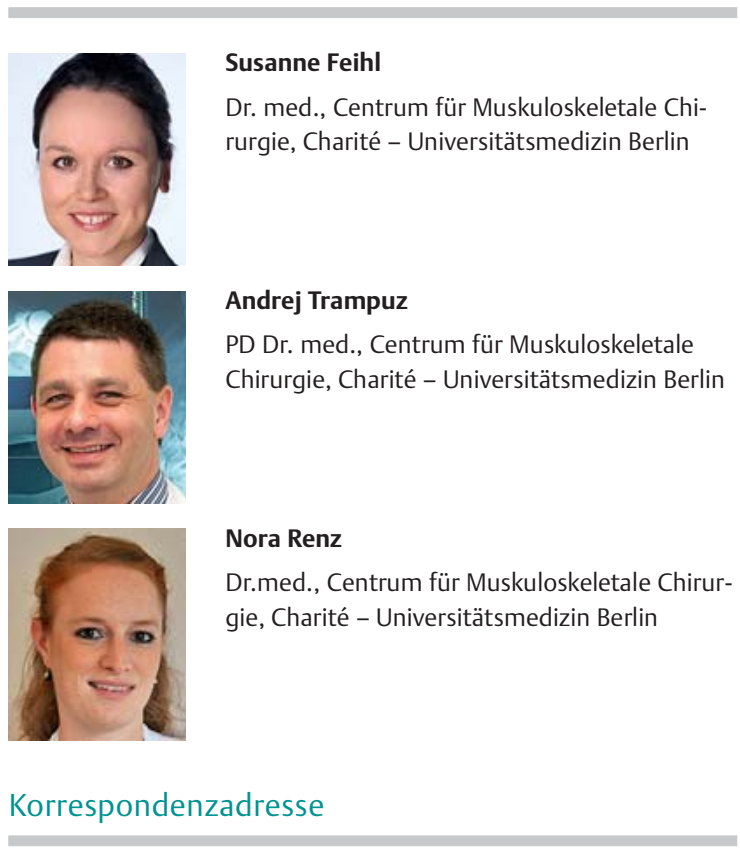

Dr. med. Susanne Feihl

Fachärztin für Mikrobiologie, Virologie und Infektionsepidemiologie Centrum für Muskuloskeletale Chirurgie Charité - Universitätsmedizin Berlin Charitéplatz 1 10117 Berlin susanne.feihl@charite.de

Literatur

[1] Achermann $Y$, Vogt $M$, Leunig $M$ et al. Improved diagnosis of periprosthetic joint infection by multiplex PCR of sonication fluid from removed implants. J Clin Microbiol 2010; 48: 1208-1214

[2] Aggarwal VK, Higuera C, Deirmengian G et al. Swab cultures are not as effective as tissue cultures for diagnosis of periprosthetic joint infection. Clin Orthop Relat Res 2013; 471: 31963203

[3] Kleber C, Schaser KD, Trampuz A. [Complication management of infected osteosynthesis: therapy algorithm for peri-implant infections]. Chirurg 2015; 86: 925-934

[4] Krenn V, Morawietz L, Kienapfel $\mathrm{H}$ et al. [Revised consensus classification. Histopathological classification of diseases associated with joint endoprostheses]. Z Rheumatol 2013; 72: 383-392

[5] Kurtz S, Ong K, Lau E et al. Projections of primary and revision hip and knee arthroplasty in the United States from 2005 to 2030. J Bone Joint Surg Am 2007; 89: 780-785 
[6] Kurtz SM, Ong KL, Lau E et al. Prosthetic joint infection risk after TKA in the Medicare population. Clin Orthop Relat Res 2010; 468: 52-56

[7] Ochsner PE, Hailemariam S. Histology of osteosynthesis associated bone infection. Injury 2006; 37 (Suppl. 2): S49-S58

[8] Portillo ME, Salvado M, Trampuz A et al. Improved diagnosis of orthopedic implant-associated infection by inoculation of sonication fluid into blood culture bottles. J Clin Microbiol 2015; 53: $1622-1627$

[9] Renz N, Cabric S, Janz V et al. [Sonication in the diagnosis of periprosthetic infections:significance and practical implementation]. Orthopade 2015; 44: 942-945

[10] Renz N, Müller M, Perka C et al. [Implant-associated infections - diagnostics]. Chirurg 2016; 87: 813-821
[11] Trampuz A, Hanssen AD, Osmon DR et al. Synovial fluid leukocyte count and differential for the diagnosis of prosthetic knee infection. Am J Med 2004; 117: 556-562

[12] Trampuz A, Piper KE, Jacobson MJ et al. Sonication of removed hip and knee prostheses for diagnosis of infection. N Engl J Med 2007; 357: 654-663

[13] Trampuz A, Zimmerli W. Diagnosis and treatment of infections associated with fracture-fixation devices. Injury 2006; 37 (Suppl. 2): S59-S66

Bibliografie

DOI https://doi.org/10.1055/s-0043-102261

OP-JOURNAL 2017; 33: 102-106 @ Georg Thieme Verlag KG Stuttgart · New York ISSN 0178-1715 